\title{
Limites e possibilidades para o cuidado em saúde à pessoa surda: perspectivas da equipe multiprofissional
}

\author{
Limits and possibilities for health care for deaf persons: perspectives of the \\ multidisciplinary team
}

Límites y posibilidades de la atención médica para personas sordas: perspectivas del equipo multidisciplinario

Cristiele Socorro de Melo ${ }^{1 *}$, Maria do Socorro Barros Said ${ }^{1}$, Marcela Milrea Araújo Barros ${ }^{1}$, Adevaldo Barroso Barbosa ${ }^{1}$, Luana Paula de Figueiredo Correia ${ }^{2}$.

\section{RESUMO}

Objetivo: Descrever os limites e possibilidades para o cuidado à pessoa surda na perspectiva da equipe multiprofissional de uma unidade de pronto atendimento. Métodos: Trata-se de um estudo investigatóriodescritivo, sustentado pela abordagem qualitativa, realizado em um um serviço de urgência e emergência localizado na região Norte do Brasil. A amostra foi composta por 12 profissionais dos quais enfermeiros, técnicos de enfermagem, médico e assistente social. Resultados: A partir da análise do conteúdo, os resultados foram agrupados em três categorias: Estratégias de comunicação da equipe com o paciente surdo no cenário da urgência e emergência, sentimentos e dificuldades vivenciadas no atendimento ao paciente surdo, e propostas para fortalecer a comunicação com o usuário surdo. A equipe refere a existência de barreiras de comunicação e utiliza como estratégias a escrita, os gestos ou a leitura labial. A capacitação multiprofissional, a presença de intérprete e a obrigatoriedade nos currículos acadêmicos da Lingua Brasileira de Sinais, são propostas que podem superar essas barreiras. Conclusão: Espera-se que este contribua com reflexões que possibilitem um olhar sensível, humanizado e integral ao usuário surdo, pautado nas suas necessidades e limitações no ambiente de cuidado em saúde.

Palavras-Chave: Equipe, Enfermagem, Atendimento, Comunicação, Surdez.

\begin{abstract}
Objective: To describe the limits and possibilities for caring for deaf people from the perspective of the multidisciplinary team of an emergency care unit. Methods: This is an investigative-descriptive study, supported by a qualitative approach, carried out in an urgent and emergency service located in the northern region of Brazil. The sample consisted of 12 professionals including nurses, nursing technicians, physicians and social workers. Results: Based on the content analysis, the results were grouped into three categories: Communication strategies of the team with deaf patients in the context of urgency and emergency, feelings and difficulties experienced in caring for deaf patients, and proposals to strengthen communication with the deaf user. The team reports the existence of communication barriers and uses writing, gestures or lip language as strategies. The multiprofessional training, the presence of an interpreter and the obligatory nature of the academic curricula of Brasilian Sing Language are proposals that can strengthen this process. Conclusion: It is expected that this will contribute with reflections that enable a sensitive, humanized and comprehensive look at the deaf user, based on their needs and limitations in the health care environment.
\end{abstract}

Keywords: Team, Nursing, Care, Communication, Deafness.

1 Faculdade Interamericana de Porto Velho - UNIRON, Porto Velho - RO.

*E-mail: crismeloromera@gmail.com

${ }^{2}$ Secretaria de Estado da Saúde de Rondônia, SESAU RO. Porto Velho - RO.

SUBMETIDO EM: 6/2021

ACEITO EM: 6/2021

PUBLICADO EM: 7/2021 


\section{RESUMEN}

Objetivo: Describir los límites y posibilidades de la atención a personas sordas desde la perspectiva del equipo multidisciplinar de una unidad de urgencias. Métodos: Se trata de un estudio investigativo-descriptivo, sustentado en un abordaje cualitativo, realizado en un servicio de urgencias y emergencias ubicado en el norte de Brasil. La muestra estuvo conformada por 12 profesionales entre enfermeros, técnicos de enfermería, médicos y trabajadores sociales. Resultados: A partir del análisis de contenido, los resultados se agruparon en tres categorías: Estrategias de comunicación del equipo con pacientes sordos en el contexto de urgencia y emergencia, sentimientos y dificultades vividas en el cuidado del paciente sordo, y propuestas para fortalecer la comunicación con los sordos. usuario. El equipo informa la existencia de barreras de comunicación y utiliza la escritura, los gestos o el lenguaje de los labios como estrategias. La formación multiprofesional, la presencia de un intérprete y la obligatoriedad de los planes de estudio académicos de Lenguaje de señas brasileño son propuestas que pueden fortalecer este proceso. Conclusión: Se espera que esto contribuya con reflexiones que permitan una mirada sensible, humanizada e integral al usuario sordo, en función de sus necesidades y limitaciones en el ámbito asistencial.

Palabras clave: Equipo, Enfermería, Atención, Comunicación, Sordera.

\section{INTRODUÇÃO}

Pessoas com deficiência ainda são estigmatizadas pela sociedade, sendo por muitas vezes reduzidas a sua deficiência, o que impede o cuidado em sua integralidade. Por esse motivo é tão importante que as legislações e políticas públicas superem seus aspectos teóricos e sejam postas em prática, ampliando espaços de discussão à população para compreensão da deficiência, das reivindicações deste grupo humano e do papel da sociedade para inclusão social destas (CORREIA LPF e SOUZA AML, 2017).

Para pessoas surdas a inclusão social deve estar relacionada sua experiência visual de mundo, e no caso de surdos usuários de língua de sinais, ocorre ao respeitar as suas peculiaridades linguísticas. É por meio da língua de sinais que esse grupo desenvolve valores e hábitos coletivos (SKLIAR C, 2013).

Em relação à inclusão de pessoas surdas nos serviços de saúde, mostra-se que as necessidades vivenciadas de usuários surdos refletem as dificuldades enfrentadas pelas pessoas com deficiência em geral. Conforme Araújo AM, et al. (2019), estes usuários são prejudicados por diversos aspectos estruturais e processuais (acessibilidade, financiamento, transporte e barreiras de comunicação) e ao acesso aos cuidados de saúde.

A Rede de Cuidados à Pessoa com Deficiência preconiza que sejam organizadas formas de atendimento inclusivo a esse público, como garantia do uso da Língua Brasileira de Sinais (BRASIL, 2012). O Decreto $5.626 / 2005$, no que tange a assistência à saúde de pessoas surdas, exige que os serviços ofertem atendimento a esse público com garantia de acessibilidade linguística, seja por meio de intérpretes de língua de sinais ou profissionais de saúde que se comuniquem em Libras (BRASIL, 2005). Contudo, as barreiras organizacionais e estruturais são enormes, principalmente no que diz respeito às fragilidades na qualificação dos profissionais em relação à comunicação, comprometendo assim, a qualidade da assistência ofertada (SOUZA MFSN, et al., 2017).

A assistência à saúde no setor de urgência e emergência tem sido utilizada de forma excessiva nos últimos anos ocasionando uma alta demanda neste setor, sendo este um importante componente no atendimento à saúde. Neste serviço, a assistência não se limita à população local, mas sim a prestação de serviços públicos sem restrições quanto ao tipo de clientela, fazendo desse serviço uma porta de entrada para os demais níveis de assistência (BRASIL, 2019).

A importância da comunicação nos serviços de urgência e emergência é fator primordial à equipe multiprofissional, já que por meio dela, se orienta o usuário surdo e o auxilia na compreensão da saúde ou de seu adoecimento, aborda os tratamentos e aponta maneiras novas e diferenciadas de comportamentos que mantenham um cuidado eficaz (VALENTE L, 2017). 
O ensino de Língua Brasileira de Sinais (LIBRAS) está instituído pelo Decreto 5.626 de 2005. Em cursos na área da saúde, o Decreto preconiza que a LIBRAS esteja inserida como disciplina curricular obrigatória apenas no curso de fonoaudiologia e de caráter optativo nos demais cursos da área de saúde (BRASIL, 2005). Durante a formação do curso superior, a aprendizagem deve estar voltada às práticas sociais do uso da linguagem em situações específicas no atendimento à saúde. Nesse cenário, a equipe multiprofissional deve buscar sempre a promoção da confiança e compreensão para os procedimentos apoio físico e emocional ao usuário do serviço, com impacto positivo no diagnóstico e tratamento (BUCKER LCG, et al., 2018).

Nas últimas décadas, como fruto dos movimentos sociais, houve um avanço das políticas públicas direcionadas a inclusão social de pessoas com deficiência, que para além de um arcabouço legal para atender esse público, passou a contar também com iniciativas advindas de políticas públicas, como o Plano Viver Sem Limites (BRASIL, 2011), o qual apresenta proposições que abrangem o acesso à educação, inclusão social, saúde e acessibilidade. Uma outra importante conquista legal para garantia de direitos foi o Estatuto da Pessoa com Deficiência (BRASIL, 2015).

É importante explorar os desafios encontrados pela equipe multiprofissional durante a prestação de serviços para surdos com vistas a fornecer soluções em prol de contornar estes problemas (PEREIRA AAC, 2020). Por isso, é fundamental que a equipe multiprofissional esteja preparada, uma vez que, a comunicação poderá permitir ao usuário maior autonomia, privacidade, atendimento individualizado, ou qualquer tipo de assistência livre de preconceitos ou barreiras (ARAÚJO AM, et al., 2019). Nessa perspectiva, a problemática exposta aponta para a seguinte questão de estudo: Quais os limites e possibilidades para o cuidado à pessoa surda no cenário da urgência e emergência? O estudo tem por objetivo descrever os limites e possibilidades para o cuidado à pessoa surda na perspectiva de profissionais de saúde de uma Unidade de Pronto Atendimento (UPA).

\section{MÉTODOS}

Trata-se de uma pesquisa de escopo investigatório e descritivo de abordagem qualitativa. O campo do estudo correspondeu a uma UPA localizada no Norte do Brasil. Foram estabelecidos como critérios de inclusão profissionais que compusessem equipe multiprofissional da unidade de saúde, de ambos os sexos, sem faixa etária definida e que atuassem em qualquer setor da UPA. Foram excluídos os profissionais em período de férias, licença ou afastamento durante o período de coleta de dados.

Salientamos que previamente a coleta de dados, o participante somente seguiria com a participação do estudo após leitura prévia e assinatura do Termo de Consentimento Livre Esclarecido (TCLE). O instrumento foi preenchido pelo próprio participante da pesquisa, em espaço reservado, garantindo o sigilo, a privacidade e a sua participaçao foi voluntária. Utilizou-se como instrumento de coleta de dados, um questionário com perguntas abertas, preenchido e recolhido com tempo aproximado de resposta de 20 a 30 minutos.

Os dados foram analisados a partir da análise de conteúdo proposta por Minayo, MCS (2010) cuja técnica se desenvolve três etapas: pré-análise, exploração do material e tratamento dos resultados. Os achados foram comparados com a literatura científica pertinente. Os participantes foram identificados pela letra maiúscula inicial da categoria profissional: Enfermeiro (E), Técnico de enfermagem (T), Médicos (M) e Assistente Social (AS) seguido pela ordem numérica de preenchimento do instrumento.

O estudo foi desenvolvido obedecendo ao estabelecido na Resolução n.. 466/2012 do Conselho Nacional de Saúde/Ministério da Saúde. Por se tratar de pesquisa com seres humanos, o estudo foi submetido ao Comitê de Ética em Pesquisa (CEP) da UNINORTE e aprovado com número CAAE: 45489421.7.0000.8028 e Parecer Consubstanciado n. 0 4.708.088 de 12 de maio de 2021.

\section{RESULTADOS E DISCUSSÃO}

A amostra final foi composta por 12 profissionais integrantes da equipe multiprofissional, dos quais haviam enfermeiros, técnicos de enfermagem, médicos e assistente social, com predominância no sexo feminino. $O$ Quadro 1, registra a caracterização dos participantes do estudo. Não havia no quadro de profissionais da UPA nutricionistas, fisioterapeutas e psicólogos. 
Quadro 1 - Caracterização da equipe multiprofissional.

\begin{tabular}{|c|c|c|}
\hline PROFISSIONAL & FAIXA ETÁRIA & SEXO \\
\hline E1 & Entre 30 a 39 anos & $\mathrm{F}$ \\
\hline E2 & Entre 27 a 29 anos & $\mathrm{F}$ \\
\hline E3 & Entre 30 a 39 anos & $\mathrm{F}$ \\
\hline E4 & Entre 30 a 39 anos & $\mathrm{F}$ \\
\hline T1 & Entre 30 a 39 anos & $\mathrm{F}$ \\
\hline T2 & Entre 30 a 39 anos & $\mathrm{F}$ \\
\hline T3 & Entre 30 a 35 anos & $\mathrm{F}$ \\
\hline T4 & Acima de 40 anos & $\mathrm{F}$ \\
\hline M1 & Entre 27 a 29 anos & $\mathrm{F}$ \\
\hline M2 & Entre 30 a 39 anos & $M^{*}$ \\
\hline M3 & Entre 30 a 39 anos & $M^{*}$ \\
\hline AS1 & Acima de 40 anos & $\mathrm{F}$ \\
\hline
\end{tabular}

Legenda: E: Enfermeiro; T: Técnico de Enfermagem; M: Médico; AS: Assistente Social; F: Feminino; ${ }^{\star}$ : Masculino. Fonte: Melo CS, et al., 2021.

A partir das análises dos dados, emergiram três categorias temáticas: Estratégias de comunicação da equipe com o paciente surdo no cenário da urgência e emergência, Sentimentos e dificuldades vivenciadas no atendimento ao paciente surdo, e propostas para fortalecer a comunicação com o usuário surdo.

\section{Estratégias de comunicação da equipe com o usuário surdo no cenário da urgência e emergência}

Nos serviços de urgência e emergência, a equipe multiprofissional desempenha um papel de extrema relevância, cabendo ao mesmo à responsabilidade e condução das ações da equipe envolvida no atendimento dos usuários que ali chegam, têm como objetivo realizar um atendimento aos usuários em estado grave. A emergência é definida como sendo a constatação de condições de agravo à saúde que impliquem em risco iminente de vida, exigindo, portanto, tratamento médico imediato (CALENZANI LD, 2014).

Diferentemente do que se assegura nas legislações a respeito da inclusão de pessoas surdas, este público não se sente respeitado em suas necessidades nos serviços de saúde e vale destacar o risco em que sua saúde e vida estão expostas, já que a dificuldade de se estabelecer uma comunicação eficiente e compreensão dos fatos pode facilmente culminar em equívocos na assistência até mesmo fatais (COSTA DGO, et al., 2021). A comunicação tende a ser o principal desafio da equipe multiprofissional no atendimento ao usuário surdo, pois há uma barreira na troca de mensagens, logo, não ocorre a compreensão de ambos os lados. À medida que o profissional de saúde não estabelece uma comunicação satisfatória, ele não identificará de forma eficiente as necessidades de saúde do usuário (SANCHES ICB, et al., 2019).

Assim, a comunicação tem um papel fundamental no processo de assistência ao usuário surdo, pois é fundamental para a interação, compreensão dos usuários, e assim descobrir suas necessidades e avaliar seus problemas de saúde. Como estratégias de comunicação implementadas no cenário da emergência ao usuário surdo, a equipe multiprofissional relata o uso de gestos, leitura labial, mímicas e português escrito:

"Leitura labial" (T3).

"Gestos e leitura labial" (AS1).

"Escrita, gestos e mímicas" (E3).

Machado WCA, et al. (2015) destacam que a equipe multiprofissional para minimizar essas dificuldades, media como configurações de comunicação com os surdos os gestos, escritas e mímicas, como pode ser detectado na fala do participante E3. Por outro lado, Britto FR e Samperiz MMF (2015) ao destacar a existência de estratégias comunicativas que podem auxiliar a equipe de saúde a se comunicar com usuário surdo, afirmam que, por muitas vezes, não funcionam de maneira eficaz.

A leitura labial em geral é sobrevalorizada pelos profissionais de saúde. Porém sua compreensão não ocorre de maneira similar entre todos os surdos. Pessoas surdas que perderam a audição após aquisição da linguagem oral podem ter mais facilidade que surdos congênitos para $O$ uso desse instrumento de comunicação (KUENBERG A, et al., 2016). Pereira AAC, et al. (2020) demonstra que apesar de ser 
frequentemente utilizada no atendimento, conforme referiram os surdos participantes da pesquisa, a leitura labial pode ser prejudicada por peculiaridades de fala, como sotaque, alteração de fluência por parte dos profissionais de saúde, ou até mesmo pelo uso de máscaras de proteção. Além disso, para Britto FR e Samperiz MMF (2015), esta estratégia requer muita atenção ao ser utilizada como forma principal de comunicação, pois diversos fonemas possuem pontos articulatórios iguais ou próximos.

Foi possível observar nas falas dos participantes que o uso do papel e caneta também correspondem como estratégias de comunicação no qual a interação acontece por meio de desenhos e escrita. Nesse caso, é importante que a equipe multiprofissional esteja atenta quanto a não utilização de terminologia técnica, buscando sempre uma linguagem mais simples e acessível ao usuário. Sujeitos surdos apontaram que essa estratégia por vezes dificulta a troca de informações, além de que o entendimento do português escrito também se relaciona com o nível de escolaridade da pessoa surda (SANTOS EM e SHIRATORE K, 2004).

Entende-se que através das estratégias de comunicação como a mímica, leitura labial, gestos e escritas, a equipe multiprofissional tenta assistir ao usuário surdo com atenção às suas necessidades de saúde, por meio de um atendimento personalizado, na tentativa de ocorrer uma comunicação e assim oferecer um serviço humanizado com resultado satisfatório. Porém a ausência e bloqueio de comunicação, que prejudica o estabelecimento de vínculo entre profissionais de saúde e surdos, compromete o atendimento e acarreta na necessidade de intermediação pelo acompanhante (PIRES HFM e ALMEIDA APT, 2016).

De acordo com estudo realizado por Oliveira YCA, et al. (2015) uma das estratégias comunicativas encontradas pela equipe de comunicar-se com a pessoa surda, é através do acompanhante que repassa a informação como tradutor para o usuário surdo, o que corrobora com a fala do participante do estudo:

"Acompanhante que saiba passar a tradução para equipe" (AS1).

Vieira CM, et al. (2017) coaduna com o exposto acima ao afirmar que é comum a presença do acompanhante na assistência à saúde. Apesar da presença frequente do acompanhante no caso de usuários surdos, em alguns casos as dúvidas de saúde não foram sanadas. Vale destacar que a necessidade de ter um acompanhante sempre que se necessite de serviço de saúde pode dificultar o acesso ao serviço tendo em vista que nem sempre há disponibilidade de acompanhante.

Para Porrozzi PS, et al. (2015), a comunicação permite aos profissionais de saúde compreenderem o usuário como um ser holístico, além de possibilitar a captação de sua visão de mundo, suas necessidades e, assim, prestar assistência de qualidade minimizando desconfortos. Os profissionais de saúde desejam cuidar dos usuários com qualidade e com o surdo não é diferente, tanto que utilizam de diversos recursos na tentativa de superar a barreira comunicacional existente.

Somente com um melhor conhecimento sobre peculiaridades comunicacionais de pessoas com deficiência auditiva, assim como a respeito da cultura, identidade, comunidade surda e da formação em Libras, haverá possibilidade de superar os entraves existentes na relação profissional de saúde e usuário surdo.

\section{Sentimentos e dificuldades vivenciados no atendimento ao paciente surdo}

A Língua Brasileira de Sinais (LIBRAS) configura-se como um formato de comunicação e expressão pensado às comunidades surdas com estrutura gramatical própria, composto por um princípio linguístico de caráter visual e motor como forma de transmissão de acontecimentos e ideias, (BRASIL, 2002). Esse formato é reconhecido como um marco histórico na luta do movimento social surdo e caracteriza-se por ser um elemento legal de comunicação desde o ano 2002.

O atendimento ao usuário surdo é um desafio para os profissionais da saúde e para o surdo, pois a comunicação por meio da fala, ou a linguagem oral, utilizada majoritariamente em nossa sociedade, não é um recurso que facilita o intercâmbio do indivíduo surdo, contrariamente, esta é uma barreira que ele precisa transcender para acessar o mundo social de forma concreta (ABREU JC, et al., 2015). Verifica-se nas narrativas dos participantes da UPA que há um misto de sentimentos contraproducentes sobre o atendimento ofertado ao usuário surdo dentre eles: incapacidade de compreensão e impotência, devido ao fato de não compreender e de se comunicar com esses pacientes:

"Incapacidade de compreensão" (T4). 


\author{
"Impotência, resulta na deficiência na prestação de um atendimento eficaz a um \\ paciente surdo/mudo" (M2).
}

No estudo de Dantas TRA, et al. (2014) a dificuldade de comunicação resulta em isolamento e, como consequência deste fato, uma assistência malsucedida ao usuário surdo. A partir das narrativas dos participantes verificam-se que esses sentimentos estão diretamente interligados as barreiras cotidianas do processo de interação. São apontadas como estratégia, a qualificação profissional para um acolhimento integral, humanizado e equânime. Através dessa comunicação constituída, o profissional pode compreender o paciente na maneira mais holística possível.

Soares IP, et al. (2018) pontuam que, ao assistir o paciente surdo nos serviços de saúde, a principal preocupação está em como se comportar diante da situação e de que maneira interagir, como comunicar-se. Nessa categoria temática emergiram como dificuldades as barreiras comunicacionais, mediante as falas mencionadas abaixo:

\title{
"Barreira comunicacional" (T1). \\ "A grande dificuldade de comunicar-se com o usuário surdo é a barreira comunicacional" (M1).
}

Segundo estudo de Reis VSL e Santos AM (2019), dentre as barreiras de acessibilidade aos surdos, destacam-se a comunicacional, que repercute negativamente no desenvolvimento dos laços requeridos na assistência à saúde, o que pode afetar o estabelecimento de diagnóstico precoce, tratamento oportuno e a adesão a terapêutica indicada. A interação entre profissional e usuário surdo é imprescindível para garantia do sucesso na assistência à saúde. É necessário que pessoas com surdez tenham seus direitos de saúde assegurados, assim como qualquer outro usuário e, desta forma, para realização de cuidado de qualidade, é necessária a compreensão de que a efetividade da comunicação é uma prioridade (SOARES IP, et al., 2018).

As dificuldades vivenciadas na relação entre a comunidade surda e sociedade, em sua maioria, como já exposto anteriormente, possuem cerne na barreira comunicacional. Entre as dificuldades encontradas os participantes relataram a falta de conhecimento de língua de sinais:

\section{"Uma das dificuldades na comunicação com o paciente surdo é a língua de sinais" (E4).}

"A língua de sinais como dificuldade na comunicação" (T4).

Costa DGO, et al. (2021) em estudo realizado com participantes surdos, apontaram a carência ou inexistência de domínio da Libras por parte dos profissionais de enfermagem, o que dificulta o atendimento holístico de pessoas com surdez na emergência hospitalar e, diante disso, suscitam a necessidade do aprendizado dessa língua para assegurar a comunicação entre os envolvidos e uma interação eficiente, relaciona intimamente com a qualidade na assistência.

Diante dos pressupostos, sabe-se que é de suma importância uma comunicação bem-sucedida entre usuário e profissional da saúde para favorecer um atendimento eficiente e humanizado. A equipe de saúde ao ter conhecimento sobre a Libras proporciona uma melhor assistência à saúde, consequentemente, há possibilidade de o surdo não precisar de intérprete ou acompanhante, proporcionando um atendimento mais humanizado, que atenda respeitando sigilo e confidencialidade. Outro ponto relevante é que o conhecimento em Libras, ao viabilizar assistência sem participação de terceiros, propicia ao surdo o protagonismo e independência no processo do cuidado a sua saúde (OLIVEIRA YCA, et al., 2015).

Diante do exposto, para inclusão social da pessoa surda no serviço de saúde é primordial que os profissionais de saúde aprendam a língua de sinais e conheçam sobre cultura e identidade surda, com vistas a qualificação da comunicação além de maior compreensão de suas necessidades, acolhimento e estabelecimento de vínculo, fundamentais no processo do cuidado.

\section{Propostas para fortalecer a comunicação com o usuário surdo}

Praticar a inclusão social implica na sociedade se adequar para receber pessoas com deficiência em seus sistemas sociais, de forma participativa, com superação de barreiras e equiparação de oportunidades 
(SASSAKI R, 2009). Neste contexto é fundamental que se discuta a temática em todos os campos, inclusive na saúde. Os entrevistados apontaram a importância das capacitações em Libras para quebrar essa barreira de comunicação, proporcionando o direito ao usuário surdo à uma assistência qualitativa e humanizada:

"Capacitação em Libras" (AS1).
"Seria bom se tivéssemos capacitação em libras para atender o paciente surdo" (T2).

"Na minha percepção seria ótimo se tivéssemos um curso de capacitação em libras" (E2).

Para amenizar problemas relacionados à assistência a pessoas surdas na saúde sugere-se que a comunicação seja direta entre surdo e profissional de saúde, mediada pela Libras. O ensino de Libras na graduação em saúde e o investimento em educação permanente para os profissionais que já atuam no serviço seria importante na viabilização da comunicação entre os envolvidos aproximando a integralidade do cuidado e respeito aos direitos das pessoas surdas (LOPES RM, et al., 2017). Vislumbra-se nesse processo a participação da pessoa surda para enriquecer o aprendizado da língua de sinais, ao trazer suas experiências, vivências, o que pode proporcionar maior engajamento por parte dos profissionais de saúde.

A pessoa com surdez, na maior parte das vezes, devido à falta de acessibilidade linguística nos serviços de saúde, utiliza-se de terceiros para intermediar a relação com os profissionais da saúde e traduzirem os conteúdos de ambas as partes durante o atendimento. Assim nas falas dos participantes, o intérprete é necessário para a comunicação com esses pacientes:

\section{"A presença do intérprete" (T1).}

Araújo AM, et al. (2019) destacaram que, conforme relatado por T1, o intérprete nas unidades de saúde pode contribuir para a realização do atendimento proporcionando acessibilidade em relação à informação entre usuários surdos e profissionais de saúde. Contudo, isso não assegura uma inclusão integral e excelente para usuários surdos. França EG, et al. (2016), descrevem que a presença do intérprete se caracteriza como medida paliativa, pois apesar de facilitar o processo comunicativo, prejudica a privacidade implicando inclusive em constrangimento ao usuário surdo por expor questões específicas de sua saúde.

Ante ao exposto, vislumbra-se como essencial que a Libras faça parte da formação dos profissionais de saúde, como disciplina obrigatória nos currículos, com vistas a inclusão da pessoa surda nessa área. Ressalta-se que a disciplina de Libras pode contribuir para a formação integral e humanizada, já que oportuniza reflexão sobre 0 respeito às diferenças, acessibilidade aos serviços, acolhimento e estabelecimento de vínculo (CORREIA LPF e SOUZA AML, 2017). Torna-se fundamental que as unidades de saúde realizem adequações estruturais para proporcionar acessibilidade a esse grupo, como sinalizações visuais, por exemplo, que invistam em educação permanente com envolvimento da temática de inclusão de surdos na saúde.

Tratar desse tema nas graduações, nos serviços de saúde, proporcionar a participação social de pessoas surdas em todos os espaços da sociedade inclusive, nas tomadas de decisões, além da responsabilidade dos governantes em pôr em prática o que já é estabelecido em Lei, possibilitará superação das problemáticas expostas nessa pesquisa. Na saúde, principalmente em serviços de urgência e emergência, o atendimento em tempo oportuno, eficaz, resolutivo e acertado faz total diferença na assistência. Um descuido, equívoco pode repercutir em desfecho desfavorável ao usuário. Agir com vistas a transformação dessa realidade é indispensável e premente.

\section{CONCLUSÃO}

Barreiras comunicações de cuidado à pessoa surda são presentes no cenário da urgência e emergência, porém, possibilidades de cuidado ao usuário surdo existem e estão ligadas à necessidade de qualificação permanente da equipe multiprofissional, presença de intérprete nos serviços de saúde e, a obrigatoriedade de um repensar à inserção de líbras como obrigatório na academia para profissionais de saúde. Espera-se que este contribua com reflexões que possibilitem um olhar sensível, humanizado e integral ao usuário surdo, pautado nas suas necessidades e limitações no ambiente de cuidado em saúde. 


\section{REFERÊNCIAS}

1. ABREU JC, et al. A percepção dos surdos em relação ao sistema de comunicação das unidades de atenção primária à saúde. Revista Baiana Enfermagem, 2015; 4(1): 10-14.

2. ARAÚJO AM, et al. A dificuldade no atendimento médico às pessoas surdas. Revista Interdisciplinar Ciências Médicas, 2019; 3(1): 3-9.

3. BUCKER LCG, et al. Comunicação Acessível na Relação Médico-Paciente Durante a Anamnese. Revista Interdisciplinar do Pensamento Científico, 2018; 4(1):133-42.

4. BRASIL. Conselho Nacional de Saúde. Resolução 466/12. Trata de pesquisas em seres humanos e atualiza a resolução 196. Diário Oficial da União. 12 dez. $2012 . \quad$ Disponível: http://www.conselho.saude.gov.br/resolucoes/2012/Reso466.pdf. Acessado em: 06 de maio de 2021.

5. BRASIL. Ministério da Saúde. Secretaria de Atenção à Saúde. Departamento de Ações Programáticas Estratégicas. Atenção à saúde da pessoa com deficiência no Sistema Unico de Saúde - SUS.1. ed. 1. reimp. - Brasília: Ministério da Saúde, 2019. Disponível em: http://bvsms.saude.gov.br/bvs/publicacoes/07_0327_M.pdf. Acessado em: 21 de junho de 2021.

6. BRASIL, Presidência da República. Decreto n. 562, de 22 de dezembro de 2005. Regulamenta a Lei $n^{\circ}$ 10.436, de 24 de abril de 2002, que dispõe sobre a Língua Brasileira de Sinais - Libras, e o art. 18 da Lei no 10.098, de 19 de dezembro de 2000. Disponível em: https://www2.camara.leg.br/legin/fed/decret/2005/decreto-5626-22-dezembro2005-539842-publicacaooriginal-39399-

pe.html\#: :text=2\%C2\%BA\%20Para\%20os\%20fins\%20deste,L\%C3\%ADngua\%20Brasileira\%20de\%20Sinais\%20\% 2D\%20Libras.

7. BRASIL. Secretaria Nacional de Promoção dos Direitos da Pessoa com Deficiência. Viver Sem Limite- Plano Nacional dos Direitos da Pessoa com Deficiência. Brasília, 2011. Disponível em: https://www.gov.br/mdh/pt-br/centrais-deconteudo/pessoa-com-deficiencia/cartilha-viver-sem-limite-plano-nacional-dos-direitos-da-pessoa-comdeficiencia/cartilha-viver-sem-limite-plano-nacional-dos.pdf

8. BRASIL. Ministério da Saúde. Lei no 13.146, de 06 de julho de 2015. Institui a Lei Brasileira de Inclusão da Pessoa com Deficiência. Diário Oficial da República Federativa do Brasil, Brasília. 2015. Disponível em: https: www//bvs.saude.gov.br/?p=3302. Acessado em: 14 junho de 2021.

9. BRASIL. Lei $N^{\circ}$ 10.436, de 24 de abril de 2002. Dispõe sobre a Língua Brasileira de Sinais - Libras e dá outras providências. Brasília, 2002. Disponível em: http://www.planalto.gov.br/ccivil_03/leis/2002//10436.htm

10. BRITTO FR, SAMPERIZ MMF. Dificuldades de comunicação e estratégias utilizadas pelos enfermeiros e sua equipe na assistência ao deficiente auditivo. Acta Paul Enferm, 2015; 8(80):5.

11. CALENZANI LD. O enfermeiro e o usuário surdo: ponto de vista prático e político, Revista Eletrônica de Enfermagem. 2017; 1 (2): 10-14.

12. CORREIA LPF, SOUZA AML. Língua de Sinais e Formação em Saúde: Uma Relação Necessária. In: CORREIA LPF, BORBA DF. (Org.). Formação, Educação e Saúde na Amazônia: (Re)significando os sentidos e práticas de saúde. 1. ed. São Carlos: Scienza, 2017. v. 1. 320p.

13. COSTA DGO, et al. A percepção de pessoas surdas sobre o acolhimento e cuidado dos profissionais de enfermagem em unidades de emergência. Revista Eletrônica Acervo Saúde, 2021; 13(5): e7451.

14. DANTAS TRA, et al. Comunicação entre a equipe de enfermagem e pessoas com deficiência auditiva. Revista Enfermagem UERJ, 2014; 22(2): 169-174.

15. FRANÇA EG, et al. Dificuldades de profissionais na atenção à saúde da pessoa com surdez severa. Ciência y enfermería, 2016; 22(3): 107-116.

16. KUENBERG A, et al. Acesso à saúde entre pessoas surdas. J Deaf Stud Deaf Educ, 2016; 21(1): 1-10.

17. LOPES RM, et al. Comunicação do surdo com profissionais de saúde na busca da integralidade. Revista Saúde e Pesquisa. 2017; 10(2): 213-221.

18. MINAYO MCS. O desafio do conhecimento. 12ª ed. São Paulo: Editora Hucitec; 2010.

19. OLIVEIRA YCA, et al. Comunicação como ferramenta essencial para assistência à saúde dos surdos. Ciência Saúde Coletiva, 2019; 25(1): 307-320.

20. PEREIRA AAC, et al. "Meu Sonho É Ser Compreendido": Uma Análise da Interação Médico-Paciente Surdo durante Assistência à Saúde. Revista Brasileira de Educação Médica, 2020; 44(4).

21. PIRES HFM, ALMEIDA APT. A percepção do surdo sobre o atendimento nos serviços de saúde. Revista Enfermagem Contemporânea, 2016; 5(1): 68-76.

22. PORROZZI PS, et al. A enfermagem e a utilização da Língua Brasileira de Sinais no atendimento ao deficiente auditivo. Revista Brasileira de Enfermagem, 2015; 17(1): 05-12.

23. REIS VSL, SANTOS AM. Conhecimento e experiência de profissionais das Equipes de Saúde da Família no atendimento a pessoas surdas. Revista Enferm UFPI, 2019; 10(2): 2-9.

24. SASSAKI RK. Inclusão: Acessibilidade no lazer, trabalho e educação. Revista Nacional de Reabilitação (Reação), 2009; Ano XII.

25. SANTOS EM, SHIRATORE K. As necessidades de saúde no mundo do silêncio: um diálogo com os surdos. Revista Eletrônica Enfermagem, 2004; 1(1).

26. SANCHES ICB, et al. O papel do enfermeiro frente ao paciente surdo, Rev enferm UFPE, 2019; 13(3): 58-62.

27. SKLIAR C. A surdez: um olhar sobre as diferenças. Porto Alegre: Editora Mediação, 2013.

28. SOARES IP, et al. Como eu falo com você? A comunicação do enfermeiro com o usuário surdo. Rev baiana enferm, 2018; 32(4).

29. SOUZA MFNS, et al. Principais dificuldades e obstáculos enfrentados pela comunidade surda no acesso à saúde: uma revisão integrativa de literatura. Rev. CEFAC, 2017. 19(3):395-405.

30. VIEIRA CM, et al. Comunicação e acessibilidade: percepções de pessoas com deficiência auditiva sobre seu atendimento nos serviços de saúde. Revista Eletrônica de Comunicação, Informação \& Invocação em Saúde, 2017; $11(2)$.

31. VALENTE L. A acessibilidade do surdo nas unidades básicas de saúde na cidade de Parintins Amazonas. Amazonas: Revista Brasileira de Enfermagem, 2017; 1(1). 